\title{
Symposium on the Crust and Upper Mantle of the Earth
}

\author{
By \\ Hisashi KunO, ${ }^{*}$ Hitoshi TAKeuCHI** and Seiya UEDA**
}

An informal symposium on the physics and chemistry of the crust and upper mantle of the earth was held on the 20th and 21st of December, 1963 at Gora, Hakone Mountains. The symposium was a continuation of two similar symposia successfully organized in 1957 and 1960 by Emeritus Professor Chuji Tsuboi of the University of Tokyo. This time, the meeting was organized by us but with the same spirit as before that an informal gathering would bring forth some very essential inter-field understanding between research workers in different fields of earth science. Such an inter-field understanding has become, in spite of it's increasing necessity, more and more difficult to acquire for anyone in these days where formal academic meetings are so tightly scheduled with an overwhelming number of highly specialized discussions. The motivation for our assembly has been to provide a remedial opportunity to this situation. Thus, following the good tradition, the symposium, being free from any external financial support, was held on a strictly personal basis at a secluded place like in Hakone Mountains. The participants of the present assembly were consisted of approximately 50 volunteers.

The topic of discussions in the afternoon of the first day was "Physical chemistry of the earch". Dr. M. Shima first spoke on the isotopic abundance ratio of various stable elements, such as $\mathrm{Li}, \mathrm{B}$ and $\mathrm{S}$ of the mantle substance and gave some evidence showing the existence of significant differences in the isotope ratios among meteorites, terrestrial rocks and mantle substances. Dr. Shima next discussed about the chemical compositions of meteorites and mantle substances using the ternary diagrams $\left(\mathrm{Na}_{2} \mathrm{O}+\right.$ $\left.\mathrm{K}_{2} \mathrm{O}\right)-\mathrm{FeO}-\mathrm{MgO}$ and $\mathrm{CaO}-\mathrm{FeO}-\mathrm{MgO}$ as usually done in the discussion of differentiation of basaltic magmas. In these talks, both the isotopic fractionation and chemical differentiation during the formation of the earth were advocated. Dr. Akimasa Masuda then introduced his unique theory on the process of formation of the earth from a molten body of meteoritic composition. The theory was based on the abundances of lanthanides in terrestrial material and meteorites. Prof. Hisashi Kuno spoke of his recent view on the origin of basaltic magmas, in particular, of possible genetical difference in basaltic magmas in island arc areas and mid-oceanic areas. Lastly Dr. Minoru Ozima made an up-to-date review on radiogenic isotope geology.

In the evening session, "Physics of the earth's upper layers" was the main topic. First, Prof. Hitoshi Takeuchi made a general survey of the recent development of the studies on mechanical properties in the earth's interior, giving special stresses on the surface wave studies and viscosity distribution. Then, seven prepared discussions followed. Dr. Tetsuo Santo reported his recent analysis of surface wave dispersion indicating regional differences in upper mantle. and crustal seismic wave velocity over the earth. Dr. Mineo Kumazawa drew attention to the possible importance of anisotropic wave velocity in rocks referring to the acutual experimental evidence. Dr. Kiyoo Mogi briefly reviewed the fracture properties of rocks under pressure at high temperature. Some difficulty was noticed for brittle fracture at great depth in the earth, which might be the case for deep focus earthquakes. Dr. Shun-iti Akimoto and Mr. Hideyuki Fujisawa gave account of their recent high pressure experiments on the olivine-spinel transformation. Prof. Tsuneji Rikitake made a review

\footnotetext{
* Department of Geology, University of Tokyo.

** Department of Geophysics, University of Tokyo.
} 
on the distribution of electrical conductivity in the upper mantle and put emphasis on its regional anomalies as revealed by electromagnetic induction phenomena. Dr. Ki-iti Hôrai discussed the recent data on the rate of radioactive heat production of rocks in relation to observed terrestrial heat flow values. Finally, Dr. Yasuo Shimazu talked about the prospect of physical approach to the geological phenomena in a general way and, as an illustration, gave a numerical analysis of tectogenesis using the sub crustal convection current as a medium of crustmantle interaction.

In the sessions of the second day, the discussion was more or less focused to the features of the "Japanese Island Arc." Dr. Tokihiko Matsuda made an essay to analyse various features characteristic to island arcs, such as zonal distribution of gravity, seismicity, heat flow, volcanic belts and other geologic structures in the light of tectonophysics. Dr. Naoto Kawai proposed, on the basis of rock magnetic observations, that the Honshu Island (Japan Proper) was twisted by about 45 degrees about vertical axis and some 15 degrees about horizontal axis at the central area in late Mesozoic time. Mr. Megumu Mizoue reported his recent analysis of geodetic data in Japan and their possible relationships with the geological structures. Dr. Seiya Uyeda gave a brief account of recent measurements of terrestrial heat flow in Japan where the heat flow was found to show a distinct zonal distribution. A mantle convection hypothesis was favoured for the interpretation. Dr. Toshi Asada, then, gave a critical summary of land explosion seismology in Japan. Among others, it was stressed that the $\mathrm{P}$-wave velocity under Moho in Japan is lower than the world's average. Dr. Sadanori Murauchi announced that very recently first successful oceanic refraction study was made in the Pacific off Kii Peninsula. Next, Dr. Shohei Banno made a quick review on the radiometric dating work on Japanese rocks. Some disagreement in the interpretation of data was found among geologists regarding the age of Sambagawa Metamorphic rocks. Returning to seismic studies, Mr. Katsusada Kaminuma depicted the crustal structure of Japan as deduced from phase velocity of Rayleigh waves and $\mathrm{Mr}$. Hiroo Kanamori spoke of his recent effort to estimate the crustal structures from seismic and gravity data in a consistent manner. Dr. Keiiti Aki, making reference to various other regions of the world, dealt with the wave velocity distribution in Japanese area and showed that under Japan the low velocity channel in the crust reaches the M-discontinuity below which the wave velocity is believed to be also lower than the normal. On the whole, general agreement seemed to hold between the opinions of these speakers. Finally, Prof. Setsumi Miyamura discussed the possible long range tectonic processes occurring during the development of the earth's crust in terms of the idea of oceanization, gravitization and such like.

Thanks to the active participation of the attendants to free discussions that followed each talk, we believe that the symposium was most stimulating throughout. We only regret that the free discussion part of the symposium can not, by it's very nature, be included in this report. 